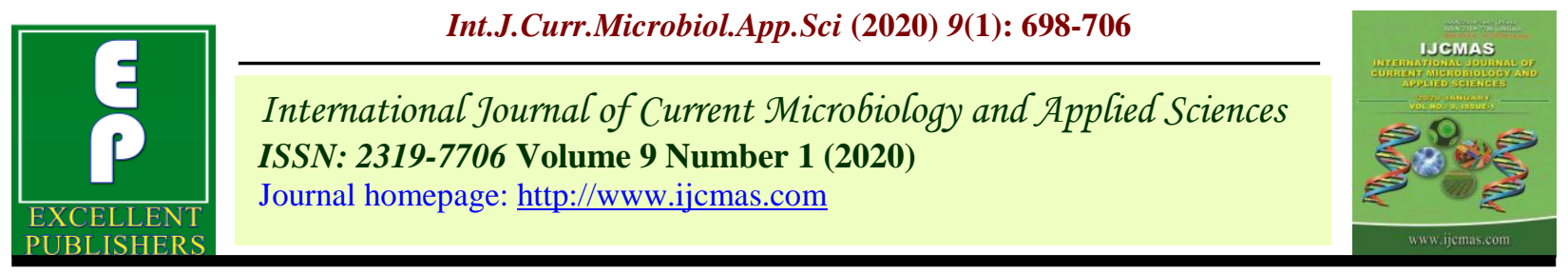

Original Research Article

https://doi.org/10.20546/ijcmas.2020.901.075

\title{
Combining Ability and Gene Action for Grain Yield and Related Components in Pearl Millet [Pennisetum glaucum (L.) R.Br.]
}

\author{
Anita Pareek*, R.V. Singh, L.D. Sharma and Shravan K. Sharma \\ Department of Genetics and Plant Breeding, Rajasthan Agriculture Research Institute, Jaipur \\ SKN Agriculture University, Jobner, Rajasthan-00000, India \\ *Corresponding author
}

Keywords

Combining ability, Gene action, Pearl millet, Line $\times$ tester analysis, Crosses, Yield

Article Info

Accepted:

15 December 2019

Available Online:

20 January 2020

\section{A B S T R A C T}

This investigation was carried out to study combining ability along with inheritance of grain yield and its component traits in 100 hybrids of pearl millet which were generated through line $\mathrm{x}$ tester mating design using 5 male sterile lines and 20 restorers as parental material at ICRISAT, Hyderabad during Summer, 2018. These hybrids were evaluated in randomized block design with 3 replications in 3 environments during Kharif, 2018 at Rajasthan Agricultural Research Institute, Durgapura (Jaipur). In results, the ratio of variance due to GCA and SCA indicated that predominance of non-additive gene action for all the characters studied except ear girth. GCA effects revealed that parents like ICMA 04999 and ICMA-843-22 (female), RIB-192, RIB-15270, RIB-494 and MIR-525-2 (male) were good general combiners for grain yield and some contributing characters. On the basis of SCA effects the crosses namely ICMA-843-22 x RIB-3135-18, ICMA-04999 x J-2290, ICMA-93333 x RIB-15270 were identified as superior for grain yield and related traits over the

\section{Introduction}

Pearl millet (Pennisetum glaucum (L.) R. Br.) is the most drought tolerant among the cultivated food grain crops. It is widely distributed across the arid and semi arid areas. Pearl millet is the fourth most important food crop after rice, wheat and sorghum. It occupies an area of 7.4 million ha in the country and Rajasthan having the biggest share 41.54 lakh ha. Its production in the country is 9.13 million tonnes and in Rajasthan it is 32.86 lakh tonnes in 2018 (Directorate of millet development). The combining ability provides better parents and desirable crosses to be used in formulation of systematic breeding and provides the nature of 
gene action involved in the inheritance of various characters. In a breeding programme, Selection of suitable parents is the most required for the success of the program. Some parents produce outstanding progenies on crossing while others, apparently equally desirable, turn out to be good combiners. This necessitates the evaluation of the parental material (inbreds) for their combining ability. The lines giving superior performance in SCA are said to be having high specific combining ability (SCA) and are best utilized in hybrid production programme.

\section{Materials and Methods}

The experimental material for present study consisted of 5 male sterile lines (ICMA93333, ICMA-843-22, ICMA-97111, ICMA04999, ICMA-94111 from The International Crops Research Institute for the Semi-Arid Tropics, Hyderabad) and 20 testers (RIB-192, RIB-494，RIB-3135-18，RIB-135071，MIR525-2, RIB-155076, RIB-155137, RIB155147, RIB-15153, RIB-15159, RIB-15181, RIB-15185， RIB-15197， RIB-15217， RIB15243, RIB-15259, RIB-15270 From RARI, Durgapura, Jaipur; J-2290, J-2340 from Junagadh Gujarat and H-77/833-2 from CCS Haryana Agriculture University). The 100 crosses were generated using line $\mathrm{x}$ tester mating design at International Crop Research Institute for the Semi-Arid Tropics (ICRISAT), Hyderabad during summer, 2018. These hybrids were grown in randomized block design with three replications in three environments created by differentiating date of sowing (the environment E1, E2 and E3 were created by early sowing, normal sowing and late sowing respectively) at Rajasthan Agricultural Research Institute (RARI), Durgapura (Jaipur) during Kharif, 2018. Each plot consisted of two rows each of 5 meter length with row spacing of $50 \mathrm{~cm}$ and plant to plant spacing of $15 \mathrm{~cm}$. All recommended cultural practices were followed to raise good crop. The observations were recorded on twelve morphological characters namely days to $50 \%$ flowering, days to maturity, plant height $(\mathrm{cm})$, number of effective tillers per plant, ear length $(\mathrm{cm})$,ear girth $(\mathrm{cm})$, flag leaf area $\left(\mathrm{cm}^{2}\right)$, dry fodder yield per plant $(\mathrm{g})$, grain yield per plant $(\mathrm{g})$, grain density $\left(\mathrm{cm}^{2}\right), 1000-$ grain weight $(\mathrm{g})$, and harvest index $(\%)$. The mean data were subjected to analyze combining ability as per the method suggested by Kempthorne (1957).

\section{Results and Discussion}

\section{Combining ability variances}

Combining ability analysis gives an idea about the magnitude of various types of gene action involved in the expression of quantitative traits for formulating an efficient breeding programme and for obtaining maximum possible gain in selection. In the present study, combining ability indicated that mean sum of squares due to crosses were significant for all the traits in all the environments (Table 1). Partitioning of these mean sum of squares due to lines were significant for all the characters except plant height, number of effective tillers and ear girth. While testers were found to be significant for most of the traits except harvest index and mean sum of squares due to interaction of males $\mathrm{x}$ females were also significant for most of the traits except plant height. Significant variance due to GCA x E for the characters indicated its sensitivity to environments. Similarly, the sensitivity of SCA to environments was observed for the traits. The ratio of variance GCA / SCA is found less than unity for all the traits except ear girth (Table 2) thereby indicating the preponderance of non-additive gene actions in the expression of the corresponding traits. SCA effects highly significant for various traits indicated epistatic gene action predominant for the characters. 
Table.1 ANOVA for combining ability for grain yield per plant and its component traits based on data

\begin{tabular}{|c|c|c|c|c|c|c|c|c|c|c|c|c|c|}
\hline \multicolumn{14}{|c|}{ Mean Squares } \\
\hline 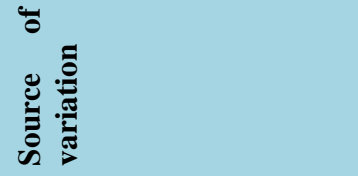 & ب் & 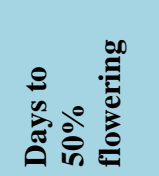 & 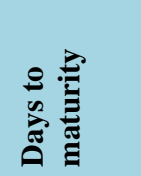 & 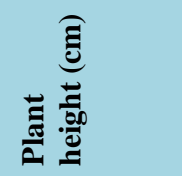 & 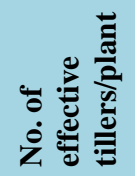 & 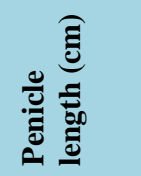 & 氖 & 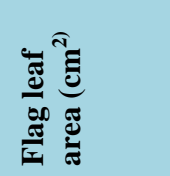 & 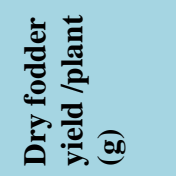 & 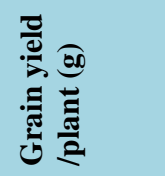 & 萢 & 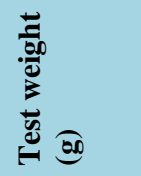 & 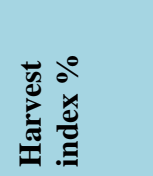 \\
\hline Replication (r) & 2 & 2.543 & 0.84 & 65.02 & 0.015 & 10.15 & 0.035 & 26.135 & 19.932 & 6.06 & 10.381 & 2.053 & 2.872 \\
\hline Environments (e) & 2 & $6984.663 * *$ & $5864.20 * *$ & $136066.621 * *$ & $1.175^{* * *}$ & $576.128 * *$ & $24.259 * *$ & $65096.465 * *$ & $75465.466^{* *}$ & $11439.732 * *$ & $1944.987 * *$ & $824.073 * *$ & $14715.708 * *$ \\
\hline Rep. $x$ Env. & 4 & 1.831 & 2.403 & 100.296 & 0.095 & 3.002 & 0.028 & 32.968 & 38.294 & $16.963^{*}$ & 12.164 & 0.743 & 30.533 \\
\hline Crosses & 99 & $13.681^{* *}$ & $11.331 * *$ & $394.722 * *$ & $0.627 * *$ & $19.826 * *$ & $0.087^{* *}$ & $1158.815 * *$ & $493.348 * *$ & $44.955 * *$ & $78.142 * *$ & $5.115 * *$ & $182.538 * *$ \\
\hline line (I) & 4 & $38.748 * *$ & $34.257 * *$ & 468.919 & 0.143 & 19.051 & $0.341 * *$ & 363.398 & $4096.089 * *$ & $219.431 * *$ & $163.213^{*}$ & $12.933 * *$ & $959.651 * *$ \\
\hline tester $(t)$ & 19 & $22.84 * *$ & $19.837 * *$ & $914.377 * *$ & $1.025^{*}$ & $62.657 * *$ & $0.243^{* *}$ & $2987.978 * *$ & $605.987 * *$ & $66.141 * *$ & $143.733 * *$ & $12.0824 * *$ & 76.179 \\
\hline $1 \times t$ & 76 & $10.071 * *$ & $7.998 * *$ & 260.903 & $0.553 * *$ & $9.159 * *$ & 0.034* & 743.388** & 275.571** & $30.475 * *$ & $57.267 * *$ & $2.962 * *$ & $168.227 * *$ \\
\hline Environment x Crosses & 198 & $7.129 * *$ & $6.252 * *$ & $283.327^{* *}$ & $0.393 * *$ & 7.949** & $0.043^{* *}$ & $737.366 * *$ & $258.224 * *$ & $22.715 * *$ & $35.300 * *$ & $3.395 * *$ & 70.572** \\
\hline Environment $x$ Line effect & 8 & $41.096 * *$ & $36.179 * *$ & 477.300* & 0.197 & $19.449 * *$ & $0.154 * *$ & $1566.751 * *$ & $1420.838 * *$ & $51.161 * *$ & $103.919 * *$ & $6.868 * *$ & 135.715 \\
\hline Environment $x$ tester effect & 38 & $9.659 * *$ & $8.340 * *$ & $566.026 * *$ & 0.327 & 19.936 ** & $0.093 * *$ & $1326.133 * *$ & $319.108 * *$ & $39.975 * *$ & $85.833 * *$ & $9.873 * *$ & 57.495 \\
\hline $\begin{array}{l}\text { Environment } x \text { Line } x \text { tester } \\
\text { effect }\end{array}$ & 152 & $4.708 * *$ & 4.155 & 202.443 & 0.420 ** & 4.348 & 0.025 & $546.522 * *$ & $181.813^{* *}$ & $16.903 * *$ & 19.056 & 1.592 & 70.412** \\
\hline Error & 594 & 4.884 & 4.378 & 213.044 & 0.066 & 4.032 & 0.026 & 272.1134 & 27.701 & 6.634 & 17.2 & 1.706 & 21.505 \\
\hline Total & 899 & 21.856 & 18.576 & $\mathbf{5 4 9 . 9 3 2}$ & 0.203 & 7.916 & 0.09 & 614.831 & 297.606 & 39.875 & 32.149 & 4.28 & 82.734 \\
\hline
\end{tabular}


Table.2 Estimates of combining ability variances for various traits over the environments

\begin{tabular}{|c|c|c|c|c|c|c|c|c|c|c|c|c|}
\hline Source of variation & $\begin{array}{c}\text { Days to } \\
\mathbf{5 0 \%} \\
\text { flowering }\end{array}$ & $\begin{array}{l}\text { Days to } \\
\text { maturity }\end{array}$ & $\begin{array}{c}\text { Plant } \\
\text { height }\end{array}$ & $\begin{array}{c}\text { No. of } \\
\text { effective } \\
\text { tillers/plant }\end{array}$ & $\begin{array}{c}\text { Ear } \\
\text { length }\end{array}$ & Ear girth & $\begin{array}{c}\text { Flag leaf } \\
\text { area }\end{array}$ & $\begin{array}{c}\begin{array}{c}\text { Dry } \\
\text { fodder } \\
\text { yield } \\
\text { /plant }\end{array} \\
\end{array}$ & $\begin{array}{c}\text { Grain } \\
\text { yield } \\
\text { /plant }\end{array}$ & $\begin{array}{c}\text { Grain } \\
\text { density }\end{array}$ & $\begin{array}{c}\text { 1000- } \\
\text { grain } \\
\text { weight }\end{array}$ & Harvest index \\
\hline$\sigma^{2} G C A$ & 0.237 & 0.208 & 4.52 & 0.004 & 0.332 & 0.002 & 12.791 & 20.668 & 1.215 & 1.226 & 0.097 & 4.435 \\
\hline$\sigma^{2} S C A$ & 0.668 & 0.486 & 8.73 & 0.051 & 0.628 & 0.001 & 56.300 & 27.746 & 2.712 & 4.643 & 0.162 & 16.586 \\
\hline$\sigma^{2} G C A / \sigma^{2} S C A$ & 0.35 & 0.43 & 0.52 & 0.08 & 0.53 & 2 & 0.23 & 0.74 & 0.45 & 0.26 & 0.6 & 0.27 \\
\hline$\sigma^{2} A$ & 0.95 & 0.832 & 18.11 & 0.01 & 1.32 & 0.009 & 51.164 & 82.673 & 4.861 & 4.906 & 0.391 & 17.740 \\
\hline$\sigma^{2} D$ & 2.67 & 1.94 & 34.94 & 0.20 & 2.51 & 0.004 & 225.203 & $\begin{array}{c}110.98 \\
4\end{array}$ & $\begin{array}{c}10.85 \\
0\end{array}$ & 18.573 & 0.651 & 66.344 \\
\hline$\sigma^{2} A / \sigma^{2} D$ & 0.36 & 0.43 & 0.52 & 0.08 & 0.52 & 2.10 & 0.227 & 0.744 & 0.448 & 0.264 & 0.601 & 0.267 \\
\hline Line (l) & 11.44 & 12.21 & 4.79 & 0.92 & 3.88 & 15.79 & 1.26 & 33.54 & 19.72 & 8.43 & 10.21 & 21.24 \\
\hline Tester $(t)$ & 32.04 & 33.59 & 44.45 & 31.34 & 60.65 & 53.65 & 49.48 & 23.57 & 28.23 & 35.3 & 45.32 & 8 \\
\hline Line $x$ Tester (lxt) & 56.51 & 54.18 & 50.74 & 67.72 & 35.46 & 30.55 & 49.24 & 42.88 & 52.04 & 56.25 & 44.45 & 70.74 \\
\hline
\end{tabular}


Table.3 Best performing parents (lines and testers) and crosses on the basis of GCA and SCA effects over the environments

\begin{tabular}{|c|c|c|c|}
\hline \multirow[t]{2}{*}{ Characters } & \multicolumn{2}{|r|}{ Parents } & \multirow[t]{2}{*}{ Crosses } \\
\hline & Lines & Testers & \\
\hline Days to $50 \%$ flowering & NS & RIB-155137, RIB-155076, J-2290 & $\begin{array}{l}\text { ICMA-843-22 x RIB-3135-18 } \\
\text { ICMA-843-22 x RIB-155137 } \\
\text { ICMA-97111 x RIB-3135-18 }\end{array}$ \\
\hline Days to maturity & ICMA-93333 & RIB-192, RIB-135076, RIB-155137, J-2290 & ICMA-94111 x RIB-3135-18 \\
\hline Plant height & ICMA-04999 & RIB-494, RIB-15153, J-2340 & $\begin{array}{l}\text { ICMA-843-22 x RIB-192 } \\
\text { ICMA-94111 x RIB-15197 }\end{array}$ \\
\hline $\begin{array}{c}\text { Number of effective } \\
\text { tillers }\end{array}$ & NS & RIB-15259, RIB-15217, H-77/833-2, RIB-494 & NS \\
\hline Ear length & ICMA-04999 & RIB-494, RIB-192, J-2340, RIB-135071, RIB-155137 & $\begin{array}{l}\text { ICMA-04999 x RIB-192 } \\
\text { NS }\end{array}$ \\
\hline Ear girth & ICMA-93333, ICMA-843-22 & H-77/833-2, RIB-155076, MIR-525-2, J-2340, RIB-135071 & $\begin{array}{l}\text { ICMA-93333 x RIB-15185 } \\
\text { ICMA-843-22 x RIB-15185 }\end{array}$ \\
\hline Flag leaf area & ICMA-843-22 & $\begin{array}{l}\text { J-2290, RIB-15185, RIB-15259, J-2340, H-77/833-2, RIB- } \\
\text { 15217, RIB-155076 }\end{array}$ & NS \\
\hline $\begin{array}{l}\text { Dry fodder yield per } \\
\text { plant }\end{array}$ & ICMA-04999 & $\begin{array}{l}\text { RIB-494, RIB-192, RIB-15270, RIB-3135-18, MIR-525-2, } \\
\text { RIB-15197, RIB-15153 }\end{array}$ & $\begin{array}{c}\text { ICMA-843-22 x RIB-192 } \\
\text { ICMA-843-22 x RIB-3135-18 } \\
\text { ICMA-94111 x RIB-494 }\end{array}$ \\
\hline Grain yield & ICMA-04999, ICMA-843-22 & RIB-192, RIB-15270, RIB-15197, RIB-494, MIR-525-2 & $\begin{array}{l}\text { ICMA-843-22 x RIB-3135-18 } \\
\text { ICMA-93333 x RIB-15270 } \\
\text { ICMA-93333 x RIB-15259 }\end{array}$ \\
\hline Grain density & ICMA-97111 & $\begin{array}{l}\text { RIB-15259, H-77/833-2, RIB-135071, RIB-155137, RIB- } \\
15191\end{array}$ & NS \\
\hline 1000- grain weight & ICMA-843-22 & $\begin{array}{l}\text { MIR-525-2, H-77/833-2, RIB-3135-18, RIB-155076, RIB- } \\
15243\end{array}$ & $\begin{array}{l}\text { ICMA-97111 x RIB-494 } \\
\text { ICMA-97111 x RIB-494 } \\
\text { ICMA-94111 x RIB-15185 }\end{array}$ \\
\hline Harvest index & $\begin{array}{l}\text { ICMA-843-22, ICMA-94111, } \\
\text { ICMA-04999 }\end{array}$ & RIB-155137, RIB-15197, RIB-15243, RIB-155076 & $\begin{array}{l}\text { ICMA-94111xRIB-15217 } \\
\text { ICMA-04999 xJ-2340 } \\
\text { ICMA-04999 x J-2290 }\end{array}$ \\
\hline
\end{tabular}


Table.4 Specific combining ability effects of best crosses for grain yield per plant and other attributes in pearl millet.

\begin{tabular}{|c|c|c|c|c|c|c|c|c|c|c|c|c|}
\hline Crosses & $\begin{array}{l}\text { Grain } \\
\text { yield }\end{array}$ & $\begin{array}{c}\text { Days to } \\
50 \% \\
\text { flowering }\end{array}$ & $\begin{array}{l}\text { Days to } \\
\text { maturity }\end{array}$ & $\begin{array}{l}\text { Plant } \\
\text { height }\end{array}$ & $\begin{array}{c}\text { Number } \\
\text { of } \\
\text { Effective } \\
\text { tillers }\end{array}$ & Ear length & $\begin{array}{l}\text { Ear } \\
\text { girth }\end{array}$ & $\begin{array}{l}\text { Flag leaf } \\
\text { area }\end{array}$ & $\begin{array}{l}\text { Dry fodder } \\
\text { yield per } \\
\text { plant }\end{array}$ & $\begin{array}{l}\text { Grain } \\
\text { density }\end{array}$ & $\begin{array}{c}\text { 1000- } \\
\text { Grain } \\
\text { weight }\end{array}$ & $\begin{array}{c}\text { Harvest } \\
\text { index }\end{array}$ \\
\hline $\begin{array}{l}\text { ICMA-843-22 x RIB- } \\
\text { 1335-18 }\end{array}$ & $3.943 * *$ & -1.216 & -0.481 & -6.280 & $0.209^{*}$ & 0.392 & -0.069 & 3.515 & $6.177 * *$ & $-2.911^{*}$ & 0.107 & 2.599 \\
\hline $\begin{array}{l}\text { ICMA- } 93333 \text { x RIB- } \\
15270\end{array}$ & $3.055^{* *}$ & $1.873 * *$ & $1.519^{*}$ & 1.931 & 0.187 & 0.942 & -0.052 & 0.469 & $3.759 *$ & 0.111 & 0.332 & 0.984 \\
\hline $\begin{array}{l}\text { ICMA-94111 x RIB- } \\
\text { 1335-18 }\end{array}$ & 0.223 & $-1.477 *$ & $-1.387 *$ & 5.470 & 0.107 & -0.443 & 0.018 & -9.122 & 1.213 & -0.778 & -0.153 & 0.622 \\
\hline $\begin{array}{l}\text { ICMA-94111 x RIB- } \\
15159\end{array}$ & $3.056^{*}$ & -0.477 & -0.120 & -0.152 & -0.001 & -0.139 & -0.072 & 1.674 & 10.834 & -2.622 & 0.098 & -2.368 \\
\hline $\begin{array}{l}\text { ICMA-94111 x H- } \\
77 / 833-2\end{array}$ & $2.034 *$ & 0.190 & 0.102 & -2.779 & $-0.207 *$ & $-2.086 * *$ & 0.065 & $-14.590 * *$ & $-5.948 * *$ & 0.244 & -0.344 & $5.629 * *$ \\
\hline $\begin{array}{l}\text { ICMA-94111 x RIB- } \\
15197\end{array}$ & 1.904* & -0.232 & -0.142 & 4.435 & $0.282 * *$ & 0.528 & 0.042 & $13.912 * *$ & $-6.546 * *$ & -1.933 & 0.449 & $4.704 * *$ \\
\hline ICMA-93333 x J-2340 & $1.5 \mathrm{I} 7$ & -1.038 & -0.570 & 5.443 & -0.137 & -0.023 & -0.004 & -4.515 & 1.484 & 0.756 & -0.035 & -1.374 \\
\hline $\begin{array}{l}\text { ICMA-843-22 x RIB- } \\
192\end{array}$ & $2.276 * *$ & $1.407^{*}$ & 1.097 & 1.931 & 0.009 & -0.563 & 0.034 & 3.450 & $10.654 * *$ & $7.178 * *$ & -0.155 & -2.339 \\
\hline $\begin{array}{l}\text { ICMA-94111 x RIB- } \\
135071\end{array}$ & $2.382 * *$ & -0.543 & -0.320 & 8.439 & -0.085 & -0.186 & 0.063 & 12.592 & 1.889 & -2.178 & 0.698 & 0.573 \\
\hline
\end{tabular}


The present findings supported by the reports of Bhardwaj et al., (2015) Mungra et al., (2015) Pawar et al., (2015) Athoni et al., (2016) Karvar et al., (2017) Badurkar et al., (2018); Gavali et al., (2018); Kumawat et al., (2019) who reported preponderance of nonadditive genetic effects. On the other hand, predominance of GCA variance or additive gene action reported by Chotaliya et al., (2010; Jethva et al., (2011) Mungra et al., (2015) Pawar et al., (2015); Krishnan et al., (2017) Gavali et al., (2018) for 1000-grain weight which are in opposition to findings of the present investigation. Such differences are expected due to differences in the experimental material and the experiments of evaluation. It is thus evident grain yield and other yield components controlled by both additive and non-additive gene effects as revealed by the combining ability analysis. However, SCA variance was more pronounced than GCA variance for all the characters except ear girth. It is therefore, suggested that the material used in the present study may be effectively utilized in the hybrid development.

The proportional contribution of lines, testers and their interaction to total variance (\%) over the environments showed maximum contribution of lines to total variance for dry fodder yield per plant (33.54\%) followed by harvest index (21.24\%). The maximum contribution of tester to total variance was for ear length $(60.65 \%)$ followed by ear girth $(53.65 \%)$. The maximum contribution to total variance in Line $\mathrm{x}$ tester interaction was displayed by harvest index $(70.74 \%)$ followed by number of effective tillers per plant $(67.72 \%)$ over the environments. Similar results were also reported by Kumar et al., (2017) and Badurkar et al., (2018).

\section{GCA and SCA effects}

The GCA and SCA effects in this section are based on the data pooled over the three environments. The best performing parents (lines and testers) and cross combinations on the basis of GCA and SCA effects (Table 3) revealed that none of the parents was found good general combiner for all the characters which suggested breeding for these characters would be effective when material is tested over a wide range of environments. The female line ICMA-04999 was found to be desirable for plant height, ear length, dry fodder yield per plant and harvest index while ICMA-843-22 was found desirable for plant ear girth, flag leaf area, 1000- grain weight and harvest index. Among males, RIB-192, RIB-15270, RIB-15270, RIB-494 and MIR525-2 exhibited significant GCA effects in over the environments for grain yield while RIB-192 exhibited significant and desirable GCA effects for ear length and dry fodder yield per plant. Male lines RIB-15270 exhibited significant and desirable GCA effects for number of effective tillers per plant and dry fodder yield per plant while RIB15197 exhibited significant and desirable GCA effects for dry fodder yield, grain density and harvest index while RIB-494 exhibited significant and desirable GCA effects for plant height, no. of effective tillers, ear length and dry fodder yield and MIR-5252 desirable for ear girth, dry fodder yield and test weight. It may be summarized that the parents ICMA-04999, ICMA-843-22 and RIB-192, RIB-15270, RIB-494, MIR-525-2 were better general combiners for grain yield per plant, dry fodder yield per plant over the environments reported earlier by Athoni et al., (2016). Krishnan et al., (2017) also reported various lines and testers, having good combining ability behavior for yield and its attributing characters in pearl millet. Top three crosses on the basis of high SCA effects for different characters are presented in Table 3.

In conclusion, the ratio of variance GCA / SCA were less then unity for all the characters except ear girth. This indicating the 
involvement non-additive gene effects in the inheritance of these characters. It may be summarized that the parents male parents RIB-192, RIB-15270, RIB-15270, RIB-494, MIR-525-2 were better general combiners and to be used as components of synthetics or composites of pearl millet. The promising crosses identified on the basis of SCA effects for seed yield per plant were ICMA-843-22 x RIB- 3135-18, ICMA-04999 x J-2290, ICMA-94111 x RIB-15159, ICMA-93333 x RIB- 15270, ICMA-93333 x RIB-15259, ICMA-97111 x RIB- 15181, ICMA-97111 x RIIB-135071, ICMA-94111 x 15185, ICMA$94111 \times$ RIB- 135071 and ICMA- 843-22 x RIB-192.

\section{References}

Athoni, B K and Boodi, I and Pattanashetti, S K and Guggari, A K (2016) Genetic diversity for yield and its component traits in pearlmillet [Pennisetum glaucum (L.) R.Br.] Int. J. of Sci.and Nature, 7 (4). pp. 795-798. ISSN 2229-6441

Badurkar, S. B., Pole, S. P., Toprope, V. N. and Ingle, N. P. 2018. Combining Ability for Grain Yield and Its Related Traits in Pearl Millet (Pennisetum glaucum) (L.) R. Br.] Int. J. Curr. Microbiol. App. Sci) Special Issue-6: 956-961

Bhardwaj, R., Kaur, M., Sohu, R., \& Singh, D. (2015). Combining Ability studies in Pearl millet [Pennisetum glaucum (L.) R. Br.]. Forage Res., 41 (2): pp. 78-84.

Chotaliya, J. M.; Dangaria, C. J. and Dhedhi, K. K. (2010). Combining ability studies in a diallel cross of ten selected restorers of pearl millet. International Journal of Agricultural Sciences, 6(1):216-219.

Gavali, R. K., Kute, N. S., Pawar, V. Y., \& Patil, H. T. (2018). Research Article Combining ability analysis and gene action studies in pearl millet [Pennisetum glaucum (L.) R Br.]. Electronic Journal of Plant Breeding, 9(3), 908-915.

Jethva, A. S.; Raval, L.; Madriya, R. B.; Mehta, D. R. And Mandavia, C. (2011). Combing ability overenvironments for grain yield and its related traits in pearl millet. Crop Improvement, 38(1): 92-96.

Karvar, S.H., Pawar, V. Y. and H. T. Patil., "Heterosis and combining ability in pearl millet", Electronic Journal of Plant Breeding 8, no. 4 (2017): 1197-1215.

Kempthorne, O. (1957). An introduction to genetic statistics: John Wiley and Sons Inc New York, pp: 458-471.

Krishnan, M. R., Patel, M. S., Gami, R. A., Bhadauria, H. S., and Patel, Y. N. (2017). Genetic analysis in pearl millet [Pennisetum glaucum (L.) R. Br.]. Int. J. Curr. Microbial. App. Sci, 6 (11), 900907.

Kumar, M., Gupta, P. C., Sharma, N., and Sharma, A. K. (2017). Estimation of standard heterosis for grain yield and yield components in pearl millet (Pennisetum glaucum (L.) R. Br.). J. of Pharmacognosy and Phytochemistry, 6(4), 785-788.

Kumawat, K.R., Gupta, P.C. and Sharma, N.K., (2019). Combining Ability and Gene Action Studies in Pearl Millet using Line $\mathrm{x}$ Tester Analysis under Arid Conditions. Int. J. Curr. Microbiol. App. Sci, 8(4), pp.976-984.

Mungra, K.S.; Dobariya, K.L. Sapovadiya, M.H. and Vavdiya, P.A, (2015). Combining ability and gene action for grain yield and its component traits in pearl millet (Pennisetum glaucum (L.) R. Br.) Electronic J. of Plant Breeding, 6(1): 66-73

Pawar, M.B., Rathod, A.H. and Patel, P.S. (2015) Combining ability effects of parents and hybrids for yield and its components in pearl millet [Pennisetum glaucum (L.) R. Br.] Green farming 6(4): 672-676. 


\section{How to cite this article:}

Anita Pareek, R.V. Singh, L.D. Sharma and Shravan K. Sharma. 2020. Combining Ability and Gene Action for Grain Yield and Related Components in Pearl Millet [Pennisetum glaucum (L.) R.Br.]. Int.J.Curr.Microbiol.App.Sci. 9(01): 698-706. doi: https://doi.org/10.20546/ijcmas.2020.901.075 